This item was submitted to Loughborough's Research Repository by the author.

Items in Figshare are protected by copyright, with all rights reserved, unless otherwise indicated.

\title{
Saturated adaptive relative motion coordination of docking ports in space close-range rendezvous
}

PLEASE CITE THE PUBLISHED VERSION

https://doi.org/10.1109/taes.2020.3003961

\section{PUBLISHER}

Institute of Electrical and Electronics Engineers (IEEE)

VERSION

AM (Accepted Manuscript)

\section{PUBLISHER STATEMENT}

Personal use of this material is permitted. Permission from IEEE must be obtained for all other uses, in any current or future media, including reprinting/republishing this material for advertising or promotional purposes, creating new collective works, for resale or redistribution to servers or lists, or reuse of any copyrighted component of this work in other works.

\section{LICENCE}

\section{All Rights Reserved}

\section{REPOSITORY RECORD}

Sun, Liang, and Jingjing Jiang. 2020. "Saturated Adaptive Relative Motion Coordination of Docking Ports in Space Close-range Rendezvous”. Loughborough University. https://hdl.handle.net/2134/12957914.v1. 


\title{
Saturated Adaptive Relative Motion Coordination of Docking Ports in Space Close-Range Rendezvous
}

\author{
Liang Sun, Member, IEEE and Jingjing Jiang, Member, IEEE
}

\begin{abstract}
An adaptive relative pose controller for docking ports of two uncertain spacecraft in autonomous rendezvous and docking is developed. A novel relative translational and rotational model represented in the chaser body-fixed frame is derived firstly based on the classical Newton-Euler equations. Based on the proposed model, a six-degrees-of-freedom adaptive control law is presented based on norm-wise estimations for the unknown parameters of two spacecraft to decrease the online computational burden. Meanwhile, an adaptive robust control input is designed by introducing an exponential function of states to improve the response performance with respect to the traditional adaptive robust control. Moreover, a linear anti-windup compensator is employed to ensure the bounded performance of the control inputs. The explicit tuning rules for designing parameters are derived based on the stability analysis of the closed-loop system. It is proved in Lyapunov framework that all closed-loop signals are always bounded and the pose tracking error ultimately converges to a small neighborhood of zero. Simulation results validate the performance of the proposed robust adaptive control strategy.
\end{abstract}

Index Terms-Space rendezvous and docking, docking ports, motion coordination, adaptive control, saturated control, model uncertainty.

\section{INTRODUCTION}

$\mathbf{M}$ ANY important space missions, such as establishment of the space station, on-orbit servicing, space debris removing, and satellite networking [1] require spacecraft rendezvous and docking technique. In the process of the in-orbit rendezvous and docking, two spacecraft, named the active chaser and the passive target, are involved in the scenario. The chaser should conduct large-angle attitude maneuvers and complicated orbital maneuvers approaching to the passive target. Specifically, the close-range rendezvous and docking operations between two spacecraft require highly accurate translational and rotational controllers, since the chaser should fly around the target to track its docking port precisely [2].

In the last few decades, many problems in rendezvous and docking missions have been considered in the control

This work was supported by the National Natural Science Foundation of China (No. 61903025), the China Scholarship Council (No. 201906465028), and the Fundamental Research Funds for the Central Universities (Nos. FRFBD-19-002A, FRF-GF-18-0028B).

L. Sun is with the Key Laboratory of Knowledge Automation for Industrial Processes of Ministry of Education, School of Automation and Electrical Engineering, University of Science and Technology Beijing, 100083 Beijing, China, and with the Institute of Artificial Intelligence, University of Science and Technology Beijing, 100083 Beijing, China. (e-mail: liangsun@ustb.edu.cn)

J. Jiang is with the Department of Aeronautical and Automotive Engineering, Loughborough University, Leicestershire, LE11 3TU, UK.(e-mail: J.Jiang2@lboro.ac.uk)

Manuscript received April XX, 20XX; revised January XX, 20XX. design. The Hamiltonian structure of the coupled attitudeorbit dynamics for orbit-attitude hovering was refined in [3]. The modeling uncertainties and unknown disturbances were handled by sliding mode control methods [4][5] and adaptive control method [6]. The optimal response performance for a flexible spacecraft close-range rendezvous was considered in controller design by using nonlinear optimal control scheme in [7]. The mass and thruster uncertainties were considered in the adaptive control system design in [8]. Collision avoidance in rendezvous and docking missions was considered in [9], and this problem was solved based on the combination of a linear quadratic regulation technique and an artificial potential function method. The complicated kinematical couplings in relative pose dynamics were considered in [10] which dealt with an element-wise adaptive state feedback control strategy. To decrease the online calculations in the adaptive controller, an integrated six-degrees-of-freedom adaptive controller was redesigned in [11]. Subsequently, to improve the pose tracking precision and decrease the transient response time, an intelligent fuzzy control approach in [12] and a robust adaptive control approach in [13] were respectively developed. To achieve the precise docking operations, a robust suboptimal control method based on differential algebra was developed in [14]. Furthermore, to achieve the integrated relative pose control, a dual quaternion-based certainty equivalence adaptive control scheme was proposed in [15]. To deal with the constrained space of the final docking cone in the rendezvous and docking missions, a dual-quaternion-based artificial potential function was proposed in [16]. Then, an adaptive state feedback controller based on the non-certainty equivalence principle was designed in [17] to enhance the control system performance.

To settle the problems of control input saturation and model uncertainties, a nonlinear disturbance observer and a linear anti-windup compensator were combined to design the controller for spacecraft rendezvous missions in [18]. Considering various system uncertainties coming from unstructured environments, a robust controller design based on the eigenstructure assignment parametric technique and the backstepping technique was developed in [19]. To address the problem of lack of relative velocity measurements, a robust observer was developed to estimate the relative velocity for rendezvous and docking controllers in [20]. Considering the short-time operation requirements of the spacecraft rendezvous and docking to save the limited fuel, an adaptive terminal sliding mode controller was designed to complete the finitetime rendezvous and proximity operations [21]. Considering the constraints on the thrust magnitude, the spacecraft positioning within the line-of-sight cone, and the approach 
velocity, the model predictive control approach [22] was employed to improve the controller real-time computational efficient [23] and satisfy the requirement of multi-objective optimization [24]. A passivity-based adaptive controller was developed in [25] for uncertain spacecraft proximity operations and a iterative-learning-control-based tracking controller was proposed in [26] for asteroid close-proximity operations, but the attitude synchronization are not considered in these studies. A time-varying sliding mode-based fault-tolerant controller was designed in [27], but the proposed model was the same as the traditional tracking model for one spacecraft so that the model couplings between the target and chaser in rendezvous and docking missions were ignored. To describe the relative pose motion between two spacecraft in a unified way, the dual quaternion-based relative pose motion model was reported recently, and some corresponding controllers were also designed, such as the non-certainty-equivalence adaptive controller in [28], output feedback controllers in [29] and [30], proportional-derivative controller in [31], and adaptive sliding mode controller in [32]. The aforementioned state feedback control approaches are able to achieve the prescribed relative pose control objectives. Nevertheless, a drawback of the above controllers is that they ignored the misalignment effect of docking ports and centers of mass for two spacecraft.

In practical applications, the chaser should fly around the target to track its docking port, and the chaser's docking port also should be aligned with the target's docking port so that the final docking operation can be conducted well. Therefore, the relative pose motion between docking ports of two spacecraft should be modeled, then the position tracking and attitude synchronization of two docking ports could be achieved in the rendezvous and docking missions. This paper gives a novel model describing the relative pose dynamics between docking ports of two spacecraft, and develops a model-based adaptive sliding mode relative pose controller for spacecraft closerange rendezvous and docking missions, where the norm-wise adaptive estimations for 8 unknown parameters in the model are updated in the proposed adaptive controller to decrease the online computational burden with respect to the classical element-wise estimations for 324 unknown parameters in [10] and 392 unknown parameters in [33]. Unlike the methods proposed in [7] and [21], the relative pose motion of the docking port-fixed coordinate frame of the chaser with respect to the target's docking port is clearly considered in the proposed relative model. The consideration of the misalignment effect of docking ports and centers of mass for two spacecraft is important, since the docking port's pose and velocities are different from the mass center's pose and velocities theoretically. Specifically, the relative pose model is formulated in the chaser's body-fixed frame to avoid the control input transformation in the traditional target's orbital coordinate framebased translational model [7] or the line-of-sight coordinate frame-based translational model [2][8]. Furthermore, although the signum function in the traditional sliding mode control generates a relatively large control chattering, the first-order sliding mode technique with an exponential reaching law and boundary layer technique is able to suppress unknown external disturbances as well as to accelerate the convergence rate towards the sliding surface and reduce the control chattering phenomenon. Moreover, the uniformly ultimately bounded stability of the closed-loop system is proved based on the Lyapunov theory, and the relative pose and velocity between two docking ports ultimately converge to small neighborhoods of zero. Simulation results have been used to validate the proposed method.

The rest of the paper is divided into four sections. Section II presents the mathematical description and control task for spacecraft rendezvous and docking. Section III provides the relative motion controller design, and the stability of the closed-loop system is rigorously analyzed. Numerical simulations are conducted in Section IV to support the theory. Conclusions and future works are discussed in Section V.

\section{Problem Statement}

Considering the docking port of the chaser tracking to the target's docking port and synchronizing the attitude of two spacecraft, some coordinate frames and vectors are defined in Figure 1, where the Earth-centered inertial frame is denoted as $\mathcal{F}_{o}=\left\{O x_{i} y_{i} z_{i}\right\}$. The vectors $\left\{\boldsymbol{r}_{t}, \boldsymbol{r}_{p}, \boldsymbol{p}_{d}\right\}$ are represented in target's body-fixed frame $\mathcal{F}_{t}=\left\{T x_{t} y_{t} z_{t}\right\}$ with an origin $\mathbf{T}$ locating at its center of mass, while the vectors $\left\{\boldsymbol{r}, \boldsymbol{r}_{e}, \boldsymbol{l}\right\}$ are described in the chaser's body-fixed frame $\mathcal{F}_{c}=\{Q x y z\}$ with an origin $\mathbf{Q}$ locating at the center of the docking port of the chaser. Point $\mathbf{C}$ is the center of mass of the chaser, and point $\mathbf{P}$ is the center position of the target's docking port. Specifically, point $\mathbf{P}$ can also be located at any position in the extension line of the target's docking port, i.e. line TP, then the proposed model and control system designing approach in this study can be extended to autonomous fly-around proximity operations.

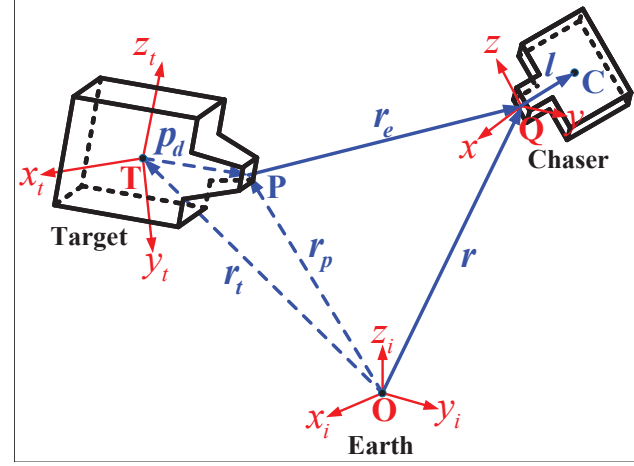

Fig. 1. Scenario of space close-range rendezvous and docking.

The position and attitude kinematics can be modeled in frame $\mathcal{F}_{c}$ as $\dot{\boldsymbol{r}}=\boldsymbol{v}-\boldsymbol{\omega} \times \boldsymbol{r}$ and $\dot{\boldsymbol{\sigma}}=G(\boldsymbol{\sigma}) \boldsymbol{\omega}$ by the modified Rodrigues parameters-based attitude description shown in [18], then the chaser's pose kinematics represented in frame $\mathcal{F}_{c}$ can be uniformly rewritten as

$$
\dot{p}=\mathcal{A p}+\mathcal{B} \boldsymbol{q}
$$

where $\boldsymbol{p}=\left[\boldsymbol{r}^{T}, \boldsymbol{\sigma}^{T}\right]^{T}, \boldsymbol{q}=\left[\boldsymbol{v}^{T}, \boldsymbol{\omega}^{T}\right]^{T}, \mathcal{A}=$ $\operatorname{diag}\left\{-S(\boldsymbol{\omega}), O_{3}\right\}, \mathcal{B}=\operatorname{diag}\left\{I_{3}, G(\boldsymbol{\sigma})\right\} ; \boldsymbol{r}$ and $\boldsymbol{v}$ are the position and velocity of frame $\mathcal{F}_{c}$ with respect to frame $\mathcal{F}_{o}$ described in frame $\mathcal{F}_{c} ; \boldsymbol{\sigma}$ and $\boldsymbol{\omega}$ are the attitude and 
angular velocity of frame $\mathcal{F}_{c}$ with respect to frame $\mathcal{F}_{o}$; $G(\boldsymbol{\sigma})=\frac{1}{4}\left[\left(1-\boldsymbol{\sigma}^{\mathrm{T}} \boldsymbol{\sigma}\right) I_{3}+2 S(\boldsymbol{\sigma})+2 \boldsymbol{\sigma} \boldsymbol{\sigma}^{\mathrm{T}}\right] ; I_{3}$ and $O_{3}$ are three-dimensional identity and null matrices, and $S(\boldsymbol{a})$ denotes a skew-symmetric matrix for any $\boldsymbol{a} \in \mathbb{R}^{3}$.

As shown in Figure 1, the position vector of mass center of the chaser can be expressed in frame $\mathcal{F}_{O}$ as

$$
\boldsymbol{r}_{c}=R_{c}(\boldsymbol{r}+\boldsymbol{l})
$$

where $\boldsymbol{l}$ is the constant position vector from the point $\mathbf{Q}$ to the mass center of the chaser $\mathbf{C}$ and $R_{c}$ is the rotation matrix from $\mathcal{F}_{c}$ to $\mathcal{F}_{o}$. Then, due to the second Newton law, the position dynamics represented in frame $\mathcal{F}_{o}$ are

$$
m \ddot{\boldsymbol{r}}_{c}=R_{c}\left(\boldsymbol{f}+\boldsymbol{f}_{g}+\boldsymbol{f}_{J_{2}}+\boldsymbol{w}\right)
$$

where $m$ is the mass of the chaser; $\boldsymbol{f}$ is the control force of the chaser; $\boldsymbol{f}_{g}$ is gravitational force [15][32]

$$
\boldsymbol{f}_{g}=m \boldsymbol{a}_{g}=-\frac{m \mu_{g}}{\left\|\boldsymbol{r}_{c}\right\|^{3}} R_{c}^{T} \boldsymbol{r}_{c}=-\frac{m \mu_{g}}{\left\|R_{c}(\boldsymbol{r}+\boldsymbol{l})\right\|^{3}}(\boldsymbol{r}+\boldsymbol{l})
$$

and $f_{J_{2}}$ is the perturbation force due to Earth's oblateness and $[15][32]$

$$
\boldsymbol{f}_{J_{2}}=m \boldsymbol{a}_{J_{2}}=-\frac{3 m \mu_{g} \mathfrak{J}_{2} \mathfrak{R}_{e}^{2}}{2\left\|R_{c}(\boldsymbol{r}+\boldsymbol{l})\right\|^{5}} R_{c}^{T}\left[\begin{array}{c}
\bar{r}_{c 1}-\frac{5 \bar{r}_{c 1}\left(\bar{r}_{c 3}\right)^{2}}{\left\|R_{c}(\boldsymbol{r}+\boldsymbol{l})\right\|^{2}} \\
\bar{r}_{c 2}-\frac{5 \bar{r}_{c 2}\left(\bar{r}_{c}\right)^{2}}{\left\|R_{c}(\boldsymbol{r}+l)\right\|^{2}} \\
5 \bar{r}_{c 3}-\frac{5\left(\bar{r}_{c 3}\right)^{3}}{\left\|R_{c}(\boldsymbol{r}+\boldsymbol{l})\right\|^{2}}
\end{array}\right]
$$

with $\bar{r}_{c i}$ representing the $i$ th element of the vector $R_{c}(\boldsymbol{r}+\boldsymbol{l})$, the second-degree zonal harmonic coefficient $\mathfrak{J}_{2}=0.0010826267$, Earth's gravitational parameter $\mu_{g}=$ $398600.4418\left(\mathrm{~km}^{3} / \mathrm{s}^{2}\right)$, and Earth's mean equatorial radius $\mathfrak{R}_{e}=6378.137(\mathrm{~km}) . \boldsymbol{w}$ represents disturbances resulting from the atmospheric drag, the solar radiation, and third-body effects [15][32].

Taking twice the time derivative of $\boldsymbol{r}_{c}$ in (2) based on the facts $\dot{R}_{c}=R_{c} S(\boldsymbol{\omega}), \dot{\boldsymbol{r}}=\boldsymbol{v}-\boldsymbol{\omega} \times \boldsymbol{r}$, and $\boldsymbol{i}=\mathbf{0}$ in frame $\mathcal{F}_{c}$ leads to

$$
\ddot{\boldsymbol{r}}_{c}=R_{c}(\boldsymbol{\omega} \times \boldsymbol{\omega} \times \boldsymbol{l}+\dot{\boldsymbol{\omega}} \times \boldsymbol{l}+\boldsymbol{\omega} \times \boldsymbol{v}+\dot{\boldsymbol{v}})
$$

Then, substituting (4) into (3) results in the position dynamics of the point $\mathbf{Q}$ as

$$
m(\dot{\boldsymbol{v}}+\dot{\boldsymbol{\omega}} \times \boldsymbol{l}+\boldsymbol{\omega} \times \boldsymbol{v}+\boldsymbol{\omega} \times \boldsymbol{\omega} \times \boldsymbol{l})=\boldsymbol{f}+\boldsymbol{f}_{g}+\boldsymbol{f}_{J_{2}}+\boldsymbol{w}
$$

Based on the Euler dynamical equation of a rigid body, the attitude motion of the chaser with respect to its mass center $\mathbf{C}$ can be modeled as

$$
J \dot{\omega}+\omega \times J \boldsymbol{\omega}=\boldsymbol{\tau}+\tau_{g}+\boldsymbol{\delta}
$$

where $J$ is the inertia matrix of the chaser with respect to $\mathbf{C}$; $\tau$ is the control torque of the chaser;

$$
\boldsymbol{\tau}_{g}=\frac{3 \mu_{g}\left(R_{c}^{T} \boldsymbol{r}_{c}\right)}{\left\|R_{c}^{T} \boldsymbol{r}_{c}\right\|^{5}} \times J\left(R_{c}^{T} \boldsymbol{r}_{c}\right)=\frac{3 \mu_{g}(\boldsymbol{r}+\boldsymbol{l})}{\|\boldsymbol{r}+\boldsymbol{l}\|^{5}} \times J(\boldsymbol{r}+\boldsymbol{l})
$$

is the gravity-gradient torque [15][32]; $\boldsymbol{\delta}$ is the unknown disturbance torque of the chaser. Then, based on the parallel axis theorem in theoretical mechanics, the inertia matrix of the chaser with respect to point $\mathbf{Q}$ is $J_{c}=J+m\left(\boldsymbol{l}^{T} \boldsymbol{l} I_{3}-\boldsymbol{l} \boldsymbol{l}^{T}\right)$, so one can obtain

$$
J_{c} \boldsymbol{\omega}=J \boldsymbol{\omega}+m \boldsymbol{l} \times \boldsymbol{\omega} \times \boldsymbol{l}=J \boldsymbol{\omega}-m \boldsymbol{l} \times \boldsymbol{l} \times \boldsymbol{\omega}
$$

based on the fact $\boldsymbol{a} \times(\boldsymbol{b} \times \boldsymbol{c})=(\boldsymbol{a} \cdot \boldsymbol{c}) \boldsymbol{b}-(\boldsymbol{a} \cdot \boldsymbol{b}) \boldsymbol{c}$ for any $\boldsymbol{a} \in \mathbb{R}^{3}, \boldsymbol{b} \in \mathbb{R}^{3}$ and $\boldsymbol{c} \in \mathbb{R}^{3}$. Taking the time derivative of $\boldsymbol{\omega}$ in (7) yields

$$
J_{c} \dot{\boldsymbol{\omega}}=J \dot{\boldsymbol{\omega}}+m \boldsymbol{l} \times(\dot{\boldsymbol{\omega}} \times \boldsymbol{l})=J \dot{\boldsymbol{\omega}}-m \boldsymbol{l} \times \boldsymbol{l} \times \dot{\boldsymbol{\omega}}
$$

Furthermore, from (5), it is derived that

$$
m \dot{\boldsymbol{\omega}} \times \boldsymbol{l}=\boldsymbol{f}+\boldsymbol{f}_{g}+\boldsymbol{f}_{J_{2}}+\boldsymbol{w}-m \dot{\boldsymbol{v}}-m \boldsymbol{\omega} \times \boldsymbol{v}-m \boldsymbol{\omega} \times \boldsymbol{\omega} \times \boldsymbol{l}
$$

and substituting (9) into (8) leads to

$J \dot{\boldsymbol{\omega}}=J_{c} \dot{\boldsymbol{\omega}}-\boldsymbol{l} \times\left(\boldsymbol{f}+\boldsymbol{f}_{g}+\boldsymbol{f}_{J_{2}}+\boldsymbol{w}-m \dot{\boldsymbol{v}}-m \boldsymbol{\omega} \times \boldsymbol{v}-m \boldsymbol{\omega} \times \boldsymbol{\omega} \times \boldsymbol{l}\right)$

Then, substituting (7) and (10) into (6) results in

$$
\begin{gathered}
J \dot{\boldsymbol{\omega}}+\boldsymbol{\omega} \times J \boldsymbol{\omega}=J_{c} \dot{\boldsymbol{\omega}}+m \boldsymbol{l} \times \dot{\boldsymbol{v}}+m \boldsymbol{l} \times \boldsymbol{\omega} \times \boldsymbol{v} \\
-\boldsymbol{l} \times\left(\boldsymbol{f}+\boldsymbol{f}_{g}+\boldsymbol{f}_{J_{2}}+\boldsymbol{w}\right)+\boldsymbol{\omega} \times J_{c} \boldsymbol{\omega}
\end{gathered}
$$

with a fact that $\boldsymbol{l} \times(\boldsymbol{\omega} \times \boldsymbol{\omega} \times \boldsymbol{l})-\boldsymbol{\omega} \times(\boldsymbol{l} \times \boldsymbol{\omega} \times \boldsymbol{l})=(\boldsymbol{\omega}$. $\boldsymbol{l})(\boldsymbol{l} \times \boldsymbol{\omega})-(\boldsymbol{\omega} \cdot \boldsymbol{\omega})(\boldsymbol{l} \times \boldsymbol{l})-(\boldsymbol{l} \cdot \boldsymbol{l})(\boldsymbol{\omega} \times \boldsymbol{\omega})+(\boldsymbol{l} \cdot \boldsymbol{\omega})(\boldsymbol{\omega} \times \boldsymbol{l})=\mathbf{0}$. Substituting (11) into (6) yields

$$
\begin{aligned}
J_{c} \dot{\boldsymbol{\omega}}+m \boldsymbol{l} \times \dot{\boldsymbol{v}} & +m \boldsymbol{l} \times \boldsymbol{\omega} \times \boldsymbol{v}+\boldsymbol{\omega} \times J_{c} \boldsymbol{\omega}=\boldsymbol{\tau}+\boldsymbol{\tau}_{g} \\
& +\boldsymbol{\delta}+\boldsymbol{l} \times\left(\boldsymbol{f}+\boldsymbol{f}_{g}+\boldsymbol{f}_{J_{2}}+\boldsymbol{w}\right)
\end{aligned}
$$

Furthermore, substituting (7) and (8) into (12) yields the attitude dynamics of the chaser represented in frame $\mathcal{F}_{c}$ as

$$
\begin{gathered}
J \dot{\boldsymbol{\omega}}-m \boldsymbol{l} \times \boldsymbol{l} \times \dot{\boldsymbol{\omega}}+m \boldsymbol{l} \times \dot{\boldsymbol{v}}+m \boldsymbol{l} \times \boldsymbol{\omega} \times \boldsymbol{v} \\
+\boldsymbol{\omega} \times J \boldsymbol{\omega}-m \boldsymbol{\omega} \times \boldsymbol{l} \times \boldsymbol{l} \times \boldsymbol{\omega}= \\
\boldsymbol{\tau}+\boldsymbol{\tau}_{g}+\boldsymbol{\delta}+\boldsymbol{l} \times\left(\boldsymbol{f}+\boldsymbol{f}_{g}+\boldsymbol{f}_{J_{2}}+\boldsymbol{w}\right)
\end{gathered}
$$

Thus the chaser's pose dynamics represented in frame $\mathcal{F}_{c}$ can be rewritten uniformly based on (5) and (13) as

$$
\mathcal{M} \dot{\boldsymbol{q}}+\mathcal{C} \boldsymbol{q}=E(\boldsymbol{u}+\boldsymbol{n}+\boldsymbol{d})
$$

where

$$
\begin{gathered}
\mathcal{M}=\left[\begin{array}{cc}
m I_{3} & -m S(\boldsymbol{l}) \\
m S(\boldsymbol{l}) & J-m S^{2}(\boldsymbol{l})
\end{array}\right], \\
\mathcal{C}=\left[\begin{array}{cc}
m S(\boldsymbol{\omega}) & -m S(\boldsymbol{\omega}) S(\boldsymbol{l}) \\
m S(\boldsymbol{l}) S(\boldsymbol{\omega}) & S(\boldsymbol{\omega}) J-m S(\boldsymbol{\omega}) S^{2}(\boldsymbol{l})
\end{array}\right], \\
E=\left[\begin{array}{cc}
I_{3} & O_{3} \\
S(\boldsymbol{l}) & I_{3}
\end{array}\right], \quad \boldsymbol{u}=\left[\begin{array}{c}
\boldsymbol{f} \\
\boldsymbol{\tau}
\end{array}\right], \\
\boldsymbol{n}=\left[\begin{array}{c}
\boldsymbol{f}_{g}+\boldsymbol{f}_{J_{2}} \\
\boldsymbol{\tau}_{g}
\end{array}\right], \quad \boldsymbol{d}=\left[\begin{array}{c}
\boldsymbol{w} \\
\boldsymbol{\delta}
\end{array}\right] .
\end{gathered}
$$

Remark 1: The matrix $\mathcal{M}$ is constant, symmetric, and positive definite, namely $\dot{\mathcal{M}}=0$ and $\mathcal{M}=\mathcal{M}^{T}>0$. Meanwhile, the matrix $\mathcal{C}$ is skew-symmetric such that $\mathcal{C}=-\mathcal{C}^{T}$, since $S(\boldsymbol{\omega}) J-m S(\boldsymbol{\omega}) S^{2}(\boldsymbol{l})=-S\left(\left(J-m S^{2}(\boldsymbol{l})\right) \boldsymbol{\omega}\right)$.

Meanwhile, the pose kinematics and dynamics of the uncontrolled target are represented, in frame $\mathcal{F}_{t}$, as

$$
\left\{\begin{array}{l}
\dot{p}_{t}=\mathcal{A}_{t} \boldsymbol{p}_{t}+\mathcal{B}_{t} \boldsymbol{q}_{t} \\
\mathcal{M}_{t} \dot{\boldsymbol{q}}_{t}+\mathcal{C}_{t} \boldsymbol{q}_{t}=\boldsymbol{d}_{t}
\end{array}\right.
$$

where $\boldsymbol{p}_{t}=\left[\boldsymbol{r}_{t}^{T}, \boldsymbol{\sigma}_{t}^{T}\right]^{T}, \quad \boldsymbol{q}_{t}=\left[\boldsymbol{v}_{t}^{T}, \boldsymbol{\omega}_{t}^{T}\right]^{T}, \mathcal{A}_{t}=$ $\operatorname{diag}\left\{-S\left(\boldsymbol{\omega}_{t}\right), O_{3}\right\}, \quad \mathcal{B}_{t}=\operatorname{diag}\left\{I_{3}, G\left(\boldsymbol{\sigma}_{t}\right)\right\}, \quad \mathcal{M}_{t}=$ $\operatorname{diag}\left\{m_{t} I_{3}, J_{t}\right\}, \quad \mathcal{C}_{t}=\operatorname{diag}\left\{m_{t} S\left(\boldsymbol{\omega}_{t}\right), S\left(\boldsymbol{\omega}_{t}\right) J_{t}\right\}, \quad \boldsymbol{d}_{t}=$ $\left[\boldsymbol{w}_{t}^{T}, \boldsymbol{\delta}_{t}^{T}\right]^{T} ; \boldsymbol{r}_{t}, \boldsymbol{v}_{t}, \boldsymbol{\sigma}_{t}, \boldsymbol{\omega}_{t}$ are the position, velocity, attitude, and angular velocity of frame $\mathcal{F}_{t}$ with respect to frame $\mathcal{F}_{o}$ described in frame $\mathcal{F}_{t} ; m_{t}$ and $J_{t}$ are the mass and the inertia 
matrix of the target; $\boldsymbol{w}_{t}$ and $\boldsymbol{\delta}_{t}$ are the external force and the external torque of the target.

To achieve the final docking missions, the position of chaser's docking port $\mathbf{Q}$ should track the position of point $\mathbf{P}$ in Figure 1. Then, the position and velocity of point $\mathbf{P}$ described in frame $\mathcal{F}_{t}$ are

$$
\boldsymbol{r}_{p}=\boldsymbol{r}_{t}+\boldsymbol{p}_{d}, \quad \boldsymbol{v}_{p}=\boldsymbol{v}_{t}+S\left(\boldsymbol{\omega}_{t}\right) \boldsymbol{p}_{d}
$$

The relative pose and velocity between points $\mathbf{Q}$ and $\mathbf{P}$ can be expressed in frame $\mathcal{F}_{c}$ as

$\boldsymbol{r}_{e}=\boldsymbol{r}-R \boldsymbol{r}_{p}, \boldsymbol{\sigma}_{e}=\boldsymbol{\sigma} \otimes \boldsymbol{\sigma}_{t}^{-1}, \boldsymbol{v}_{e}=\boldsymbol{v}-R \boldsymbol{v}_{p}, \boldsymbol{\omega}_{e}=\boldsymbol{\omega}-R \boldsymbol{\omega}_{t}$

where $\boldsymbol{\sigma} \otimes \boldsymbol{\sigma}_{t}^{-1}=\frac{\boldsymbol{\sigma}_{t}\left(\boldsymbol{\sigma}^{\mathrm{T}} \boldsymbol{\sigma}-1\right)+\boldsymbol{\sigma}\left(1-\boldsymbol{\sigma}_{t}^{\mathrm{T}} \boldsymbol{\sigma}_{t}\right)-2 S\left(\boldsymbol{\sigma}_{t}\right) \boldsymbol{\sigma}}{1+\boldsymbol{\sigma}_{t}^{\mathrm{T}} \boldsymbol{\sigma}_{t} \boldsymbol{\sigma}^{\mathrm{T}} \boldsymbol{\sigma}+2 \boldsymbol{\sigma}_{t}^{\mathrm{T}} \boldsymbol{\sigma}}, R$ is the rotational matrix from $\mathcal{F}_{t}$ to $\mathcal{F}_{c}$ and is expressed as [34]

$$
R=I_{3}-\frac{4\left(1-\boldsymbol{\sigma}_{e}^{\mathrm{T}} \boldsymbol{\sigma}_{e}\right)}{\left(1+\boldsymbol{\sigma}_{e}^{\mathrm{T}} \boldsymbol{\sigma}_{e}\right)^{2}} S\left(\boldsymbol{\sigma}_{e}\right)+\frac{8}{\left(1+\boldsymbol{\sigma}_{e}^{\mathrm{T}} \boldsymbol{\sigma}_{e}\right)^{2}} S^{2}\left(\boldsymbol{\sigma}_{e}\right) .
$$

Furthermore, based on the fact that $\dot{R}=-S\left(\boldsymbol{\omega}_{e}\right) R$, calculating the time derivative of relative pose and velocity results in the relative kinematics and relative dynamics expressed in frame $\mathcal{F}_{c}$ as

$$
\left\{\begin{array}{l}
\dot{\boldsymbol{p}}_{e}=\mathcal{A}_{e} \boldsymbol{p}_{e}+\mathcal{B}_{e} \boldsymbol{q}_{e} \\
\mathcal{M}_{e} \dot{\boldsymbol{q}}_{e}+\mathcal{C}_{e} \boldsymbol{q}_{e}+\boldsymbol{g}_{e}=E \boldsymbol{u}+\boldsymbol{d}_{e}
\end{array}\right.
$$

where $\boldsymbol{p}_{e}=\left[\boldsymbol{r}_{e}, \boldsymbol{\sigma}_{e}^{T}\right]^{T}, \boldsymbol{q}_{e}=\left[\boldsymbol{v}_{e}^{T}, \boldsymbol{\omega}_{e}^{T}\right]^{T}, \mathcal{A}_{e}=\mathcal{A}, \mathcal{B}_{e}=$ $\operatorname{diag}\left\{I_{3}, G\left(\boldsymbol{\sigma}_{e}\right)\right\}, \mathcal{M}_{e}=\mathcal{M}, \mathcal{C}_{e}=\mathcal{C}, \boldsymbol{g}_{e}=\left[\boldsymbol{g}_{e 1}^{T}, \boldsymbol{g}_{e 2}^{T}\right]^{T}, \boldsymbol{d}_{e}=$ $\left[\boldsymbol{d}_{e 1}^{T}, \boldsymbol{d}_{e 2}^{T}\right]^{T}$, and

$$
\begin{aligned}
\boldsymbol{g}_{e 1}= & m\left[S(\boldsymbol{l}) S\left(\boldsymbol{\omega}_{e}\right)-S(\boldsymbol{\omega}) S(\boldsymbol{l})\right]\left(\boldsymbol{\omega}-\boldsymbol{\omega}_{e}\right)+m[S(\boldsymbol{l}) R+ \\
& \left.R S\left(\boldsymbol{p}_{d}\right)\right] J_{t}^{-1} S\left(R^{T}\left(\boldsymbol{\omega}-\boldsymbol{\omega}_{e}\right)\right) J_{t} R^{T}\left(\boldsymbol{\omega}-\boldsymbol{\omega}_{e}\right)+ \\
& m S^{2}\left(\boldsymbol{\omega}-\boldsymbol{\omega}_{e}\right) R \boldsymbol{p}_{d}+m R S^{2}\left(R^{T}\left(\boldsymbol{\omega}-\boldsymbol{\omega}_{e}\right)\right) \boldsymbol{p}_{d}- \\
& \boldsymbol{f}_{g}-\boldsymbol{f}_{J_{2}}, \\
\boldsymbol{g}_{e 2}= & m S(\boldsymbol{l}) S^{2}\left(\boldsymbol{\omega}-\boldsymbol{\omega}_{e}\right) R \boldsymbol{p}_{d}-\left[J R-m S(\boldsymbol{l}) R S\left(\boldsymbol{p}_{d}\right)-\right. \\
& \left.m S^{2}(\boldsymbol{l}) R\right] J_{t}^{-1} S\left(R^{T}\left(\boldsymbol{\omega}-\boldsymbol{\omega}_{e}\right)\right) J_{t} R^{T}\left(\boldsymbol{\omega}-\boldsymbol{\omega}_{e}\right)+ \\
& {\left[m S^{2}(\boldsymbol{l}) S\left(\boldsymbol{\omega}_{e}\right)-m S(\boldsymbol{\omega}) S^{2}(\boldsymbol{l})+S(\boldsymbol{\omega})-\right.} \\
& \left.J S\left(\boldsymbol{\omega}_{e}\right)\right]\left(\boldsymbol{\omega}-\boldsymbol{\omega}_{e}\right)+m S(\boldsymbol{l}) R S^{2}\left(R^{T}(\boldsymbol{\omega}-\right. \\
& \left.\left.\boldsymbol{\omega}_{e}\right)\right) \boldsymbol{p}_{d}-\boldsymbol{\tau}_{g}-S(\boldsymbol{l})\left(\boldsymbol{f}_{g}+\boldsymbol{f}_{J_{2}}\right), \\
\boldsymbol{d}_{e 1}= & \boldsymbol{w}-\frac{m}{m_{t}} R \boldsymbol{w}_{t}+m\left[S(\boldsymbol{l}) R+R S\left(\boldsymbol{p}_{d}\right)\right] J_{t}^{-1} \boldsymbol{\delta}_{t}, \\
\boldsymbol{d}_{e 2}= & \boldsymbol{\delta}+S(\boldsymbol{l}) \boldsymbol{w}-\frac{m}{m_{t}} S(\boldsymbol{l}) R \boldsymbol{w _ { t }}-[J R- \\
& \left.m S(\boldsymbol{l}) R S\left(\boldsymbol{p}_{d}\right)-m S^{2}(\boldsymbol{l}) R\right] J_{t}^{-1} \boldsymbol{\delta}_{t} .
\end{aligned}
$$

Consider that the chaser's inputs $\boldsymbol{f}=\left[f_{1}, f_{2}, f_{3}\right]^{\mathrm{T}}$ and $\boldsymbol{\tau}=\left[\tau_{1}, \tau_{2}, \tau_{3}\right]^{\mathrm{T}}$ are subject to the following nonsymmetric constraints [18]

$$
-f_{i_{\min }} \leq f_{i} \leq f_{i_{\max }},-\tau_{i_{\min }} \leq \tau_{i} \leq \tau_{i_{\max }}, i=1,2,3
$$

where $f_{i_{\min }}$ and $\tau_{i_{\min }}$ are the known lower limitations of inputs, $f_{i_{\max }}$ and $\tau_{i_{\max }}$ are the known upper limitations of inputs. Thus, the control inputs $f_{i}$ and $\tau_{i}$ are defined by

$$
f_{i}= \begin{cases}f_{i_{\max }} & \text { if } f_{i_{0}}>f_{i_{\max }} \\ f_{i_{0}} & \text { if }-f_{i_{\min }} \leq f_{i_{0}} \leq f_{i_{\max }} \\ -f_{i_{\min }} & \text { if } f_{i_{0}}<-f_{i_{\min }}\end{cases}
$$

$$
\tau_{i}= \begin{cases}\tau_{i_{\max }} & \text { if } \tau_{i_{0}}>\tau_{i_{\max }} \\ \tau_{i_{0}} & \text { if }-\tau_{i_{\min }} \leq \tau_{i_{0}} \leq \tau_{i_{\max }} \\ -\tau_{i_{\min }} & \text { if } \tau_{i_{0}}<-\tau_{i_{\min }}\end{cases}
$$

where $\boldsymbol{f}_{0}=\left[f_{1_{0}}, f_{2_{0}}, f_{3_{0}}\right]^{\mathrm{T}}$ and $\boldsymbol{\tau}_{0}=\left[\tau_{1_{0}}, \tau_{2_{0}}, \tau_{3_{0}}\right]^{\mathrm{T}}$ are the control input commands to be designed.

Before the statement of the control objective of this study, the following assumptions are claimed.

Assumption 1: $m$ and $J$ are uncertain but constant, while $m_{t}$ and $J_{t}$ are completely unknown but constant. Furthermore, the chaser's parameters can be treated as $m=m_{0}+m_{\Delta}$ and $J=J_{0}+J_{\Delta}$ with the known constant parts $m_{0}$ and $J_{0}$ and the unknown parts $m_{\Delta}$ and $J_{\Delta}$. The external disturbances are unknown and bounded by constants $\|\boldsymbol{d}\| \leq \bar{d}$ and $\left\|\boldsymbol{d}_{t}\right\| \leq \bar{d}_{t}$, respectively. The desired position vector $\boldsymbol{p}_{d}$ and the vector $\boldsymbol{l}$ are constant and known by the chaser. Moreover, the influence of orbital motion of two spacecraft are excluded here since the motion of spacecraft is largely faster than that of orbital motion.

Assumption 2: The chaser is active for rendezvous and docking with a passive target in this study, while the chaser can obtained precisely its motion information $\boldsymbol{p}$ and $\boldsymbol{q}$ and relative motion information $\boldsymbol{p}_{e}$ and $\boldsymbol{q}_{e}$ in real time based on the highperformance measurement devices and equipments which are mounted on the body of the chaser.

This paper aims to develop an adaptive controller $\boldsymbol{u}$ under the Assumptions 1 and 2 to ensure the precisely relative position tracking and attitude synchronization between two docking ports of two spacecraft, while system states $\boldsymbol{p}_{e}(t)$ and $\boldsymbol{q}_{e}(t)$ ultimately converge to small neighborhoods of zero.

\section{Controller Design And Stability Analysis}

Define a vector

$$
s=\boldsymbol{q}_{e}+\Lambda \boldsymbol{p}_{e}
$$

with a positive-definite diagonal gain matrix $\Lambda=$ $\operatorname{diag}\left\{\Lambda_{1}, \Lambda_{2}\right\}$ and $\Lambda_{i} \in \mathbb{R}^{3 \times 3}>0$. Then, based on (18) and (22), the time derivative of $s$ can be obtained by

$$
\begin{aligned}
\mathcal{M}_{e} \dot{\boldsymbol{s}}+\mathcal{C}_{e} s= & \mathcal{M}_{e} \Lambda \mathcal{B}_{e} \boldsymbol{q}_{e}+\mathcal{M}_{e} \Lambda \mathcal{A}_{e} \boldsymbol{p}_{e}+ \\
& \mathcal{C}_{e} \Lambda \boldsymbol{p}_{e}-\boldsymbol{g}_{e}+E \boldsymbol{u}+\boldsymbol{d}_{e}
\end{aligned}
$$

Furthermore, because of the parametric uncertainties of $m$ and $J$ in Assumption 1, the matrices can be written as $\mathcal{M}_{e}=$ $\mathcal{M}_{0}+\mathcal{M}_{\Delta}, \mathcal{C}_{e}=\mathcal{C}_{0}+\mathcal{C}_{\Delta}$, and $\boldsymbol{g}_{e}=\boldsymbol{g}_{0}+\boldsymbol{g}_{\Delta}$, then the dynamics of $s$ can be rewritten as

$$
\mathcal{M}_{e} \dot{\boldsymbol{s}}+\mathcal{C}_{e} \boldsymbol{s}=\boldsymbol{h}_{0}+\boldsymbol{h}_{\Delta}+E \boldsymbol{u}_{0}+E \boldsymbol{u}_{\Delta}+\boldsymbol{d}_{e}
$$

where

$$
\begin{gathered}
\boldsymbol{h}_{0}=\mathcal{M}_{0} \Lambda\left(\mathcal{A}_{e} \boldsymbol{p}_{e}+\mathcal{B}_{e} \boldsymbol{q}_{e}\right)+\mathcal{C}_{0} \Lambda \boldsymbol{p}_{e}-\boldsymbol{g}_{0}, \\
\boldsymbol{h}_{\Delta}=\mathcal{M}_{\Delta} \Lambda\left(\mathcal{A}_{e} \boldsymbol{p}_{e}+\mathcal{B}_{e} \boldsymbol{q}_{e}\right)+\mathcal{C}_{\Delta} \Lambda \boldsymbol{p}_{e}-\boldsymbol{g}_{\Delta}, \\
\mathcal{M}_{0}=\left[\begin{array}{cc}
m_{0} I_{3} & -m_{0} S(\boldsymbol{l}) \\
m_{0} S(\boldsymbol{l}) & J_{0}-m_{0} S^{2}(\boldsymbol{l})
\end{array}\right], \\
\mathcal{M}_{\Delta}=\left[\begin{array}{cc}
m_{\Delta} I_{3} & -m_{\Delta} S(\boldsymbol{l}) \\
m_{\Delta} S(\boldsymbol{l}) & J_{\Delta}-m_{\Delta} S^{2}(\boldsymbol{l})
\end{array}\right], \\
\mathcal{C}_{0}=\left[\begin{array}{cc}
m_{0} S(\boldsymbol{\omega}) & -m_{0} S(\boldsymbol{\omega}) S(\boldsymbol{l}) \\
m_{0} S(\boldsymbol{l}) S(\boldsymbol{\omega}) & S(\boldsymbol{\omega}) J_{0}-m_{0} S(\boldsymbol{\omega}) S^{2}(\boldsymbol{l})
\end{array}\right],
\end{gathered}
$$




$$
\begin{aligned}
& \mathcal{C}_{\Delta}= {\left[\begin{array}{cc}
m_{\Delta} S(\boldsymbol{\omega}) & -m_{\Delta} S(\boldsymbol{\omega}) S(\boldsymbol{l}) \\
m_{\Delta} S(\boldsymbol{l}) S(\boldsymbol{\omega}) & S(\boldsymbol{\omega}) J_{\Delta}-m_{\Delta} S(\boldsymbol{\omega}) S^{2}(\boldsymbol{l})
\end{array}\right] } \\
& \boldsymbol{g}_{0}=\left[\boldsymbol{g}_{01}^{T}, \boldsymbol{g}_{02}^{T}\right]^{T}, \boldsymbol{g}_{\Delta}=\left[\boldsymbol{g}_{\Delta 1}^{T}, \boldsymbol{g}_{\Delta 2}^{T}\right]^{T} \\
& \boldsymbol{g}_{01}=m_{0}\left[S(\boldsymbol{l}) S\left(\boldsymbol{\omega}_{e}\right)-S(\boldsymbol{\omega}) S(\boldsymbol{l})\right]\left(\boldsymbol{\omega}-\boldsymbol{\omega}_{e}\right)+m_{0} S^{2}(\boldsymbol{\omega}- \\
&\left.\boldsymbol{\omega}_{e}\right) R \boldsymbol{p}_{d}+m_{0} R S^{2}\left(R^{T}\left(\boldsymbol{\omega}-\boldsymbol{\omega}_{e}\right)\right) \boldsymbol{p}_{d}-m_{0}\left(\boldsymbol{a}_{g}+\boldsymbol{a}_{J_{2}}\right), \\
& \boldsymbol{g}_{02}=m_{0} S(\boldsymbol{l}) S^{2}\left(\boldsymbol{\omega}-\boldsymbol{\omega}_{e}\right) R \boldsymbol{p}_{d}+\left[m_{0} S^{2}(\boldsymbol{l}) S\left(\boldsymbol{\omega}_{e}\right)-\right. \\
&\left.m_{0} S(\boldsymbol{\omega}) S^{2}(\boldsymbol{l})+S(\boldsymbol{\omega})-J_{0} S\left(\boldsymbol{\omega}_{e}\right)\right]\left(\boldsymbol{\omega}-\boldsymbol{\omega}_{e}\right)+ \\
& m_{0} S(\boldsymbol{l}) R S^{2}\left(R^{T}\left(\boldsymbol{\omega}-\boldsymbol{\omega}_{e}\right)\right) \boldsymbol{p}_{d} \\
&-\boldsymbol{\tau}_{g 0}-m_{0} S(\boldsymbol{l})\left(\boldsymbol{a}_{g}+\boldsymbol{a}_{J_{2}}\right) \\
& \boldsymbol{g}_{\Delta 1}= m_{\Delta}\left[S(\boldsymbol{l}) S\left(\boldsymbol{\omega}_{e}\right)-S(\boldsymbol{\omega}) S(\boldsymbol{l})\right]\left(\boldsymbol{\omega}-\boldsymbol{\omega}_{e}\right)+ \\
& m\left[S(\boldsymbol{l}) R+R S\left(\boldsymbol{p}_{d}\right)\right] J_{t}^{-1} S\left(R^{T}\left(\boldsymbol{\omega}-\boldsymbol{\omega}_{e}\right)\right) J_{t} R^{T}(\boldsymbol{\omega}- \\
&\left.\boldsymbol{\omega}_{e}\right)+m_{\Delta} S^{2}\left(\boldsymbol{\omega}-\boldsymbol{\omega}_{e}\right) R \boldsymbol{p}_{d}+m_{\Delta} R S^{2}\left(R^{T}(\boldsymbol{\omega}-\right. \\
&\left.\left.\boldsymbol{\omega}_{e}\right)\right) \boldsymbol{p}_{d}-m_{\Delta}\left(\boldsymbol{a}_{g}+\boldsymbol{a}_{J_{2}}\right) \\
& \boldsymbol{g}_{\Delta 2}= m_{\Delta} S(\boldsymbol{l}) S^{2}\left(\boldsymbol{\omega}-\boldsymbol{\omega}_{e}\right) R \boldsymbol{p}_{d}-\left[J R-m S(\boldsymbol{l}) R S\left(\boldsymbol{p}_{d}\right)-\right. \\
&\left.m S^{2}(\boldsymbol{l}) R\right] J_{t}^{-1} S\left(R^{T}\left(\boldsymbol{\omega}-\boldsymbol{\omega}_{e}\right)\right) J_{t} R^{T}\left(\boldsymbol{\omega}-\boldsymbol{\omega}_{e}\right)+ \\
& {\left[m_{\Delta} S^{2}(\boldsymbol{l}) S\left(\boldsymbol{\omega}_{e}\right)-m_{\Delta} S(\boldsymbol{\omega}) S^{2}(\boldsymbol{l})+S(\boldsymbol{\omega})-\right.} \\
&\left.J_{\Delta} S\left(\boldsymbol{\omega}_{e}\right)\right]\left(\boldsymbol{\omega}-\boldsymbol{\omega}_{e}\right)+m_{\Delta} S(\boldsymbol{l}) R S^{2}\left(R^{T}(\boldsymbol{\omega}-\right. \\
&\left.\left.\boldsymbol{\omega}_{e}\right)\right) \boldsymbol{p}_{d}-\boldsymbol{\tau}_{g \Delta}-m_{\Delta} S(\boldsymbol{l})\left(\boldsymbol{a}_{g}+\boldsymbol{a}_{J_{2}}\right)
\end{aligned}
$$

with $\boldsymbol{\tau}_{g 0}=\frac{3 \mu_{g}(\boldsymbol{r}+\boldsymbol{l})}{\|\boldsymbol{r}+\boldsymbol{l}\|^{5}} \times J_{0}(\boldsymbol{r}+\boldsymbol{l}), \boldsymbol{\tau}_{g \Delta}=\frac{3 \mu_{g}(\boldsymbol{r}+\boldsymbol{l})}{\|\boldsymbol{r}+\boldsymbol{l}\|^{5}} \times J_{\Delta}(\boldsymbol{r}+\boldsymbol{l})$, $\boldsymbol{u}_{\Delta}=\boldsymbol{u}-\boldsymbol{u}_{0}$ and $\boldsymbol{u}_{0}=\left[\boldsymbol{f}_{0}^{T}, \boldsymbol{\tau}_{0}^{T}\right]^{T}$.

Defining eight unknown constant parameters as

$$
\begin{aligned}
\alpha_{1}= & \left\|\mathcal{M}_{\Delta}\right\|, \\
\alpha_{2}= & \left|m_{\Delta}\right|+2\left|m_{\Delta}\right|\|\boldsymbol{l}\|+\left\|J_{\Delta}\right\|+\left|m_{\Delta}\right|\|\boldsymbol{l}\|^{2}, \\
\alpha_{3}= & \left(\|\boldsymbol{l}\|+\left\|\boldsymbol{p}_{d}\right\|\right) m\left\|J_{t}^{-1}\right\|\left\|J_{t}\right\|+4\left|m_{\Delta}\right|\left\|\boldsymbol{p}_{d}\right\|+ \\
& \left(\|J\|+m\|\boldsymbol{l}\|\left\|\boldsymbol{p}_{d}\right\|+m\|\boldsymbol{l}\|^{2}\right)\left\|J_{t}^{-1}\right\|\left\|J_{t}\right\|, \\
\alpha_{4}= & \left|m_{\Delta}\right|\|\boldsymbol{l}\|+\left|m_{\Delta}\right|\|\boldsymbol{l}\|^{2}+\left\|J_{\Delta}\right\|, \\
\alpha_{5}= & 1+\left|m_{\Delta}\right|\|\boldsymbol{l}\|+\left|m_{\Delta}\right|\|\boldsymbol{l}\|^{2}, \\
\alpha_{6}= & \left|m_{\Delta}\right|+\left|m_{\Delta}\right|\|\boldsymbol{l}\|, \alpha_{7}=\left\|J_{\Delta}\right\|, \\
\alpha_{8}= & {\left[(1+\|\boldsymbol{l}\|) \frac{m}{m_{t}}+(m+1)\left\|\boldsymbol{p}_{d}\right\|+2 m\|\boldsymbol{l}\|+\right.} \\
& \left.\|J\|+m\|\boldsymbol{l}\|^{2}\right]\left\|J_{t}^{-1}\right\| \bar{d}_{t}+(1+\|\boldsymbol{l}\|) \bar{d},
\end{aligned}
$$

and their estimations as $\hat{\alpha}_{i}(i=1,2, \cdots, 8)$, then one can design a model-based adaptive controller as

$$
\boldsymbol{u}_{0}=E^{-1}\left[-k \mathcal{B}_{e}^{T} \boldsymbol{p}_{e}-K \boldsymbol{s}-L \boldsymbol{\xi}-\boldsymbol{h}_{0}-\frac{\gamma}{\beta(\boldsymbol{s})} \tanh (\kappa \boldsymbol{s})\right]
$$

with $\gamma=\sum_{i=1}^{8} \hat{\alpha}_{i} \zeta_{i}, \zeta_{1}=\left\|\Lambda\left(\mathcal{A}_{e} \boldsymbol{p}_{e}+\mathcal{B}_{e} \boldsymbol{q}_{e}\right)\right\|, \zeta_{2}=$ $\|\boldsymbol{\omega}\|\left\|\Lambda \boldsymbol{p}_{e}\right\|, \zeta_{3}=\left\|\boldsymbol{\omega}-\boldsymbol{\omega}_{e}\right\|^{2}, \zeta_{4}=\left\|\boldsymbol{\omega}_{e}\right\|\left\|\boldsymbol{\omega}-\boldsymbol{\omega}_{e}\right\|, \zeta_{5}=$ $\|\boldsymbol{\omega}\|\left\|\boldsymbol{\omega}-\boldsymbol{\omega}_{e}\right\|, \zeta_{6}=\left\|\boldsymbol{a}_{g}+\boldsymbol{a}_{J_{2}}\right\|, \zeta_{7}=\frac{3 \mu_{g}}{\|\boldsymbol{r}+\boldsymbol{l}\|^{3}}, \zeta_{8}=1$, and design the adaptive laws as

$$
\dot{\hat{\alpha}}_{i}=-\rho_{i} \hat{\alpha}_{i}+\frac{\lambda_{i} \zeta_{i}}{\beta(s)} \boldsymbol{s}^{T} \tanh (\kappa \boldsymbol{s}),(i=1,2, \cdots, 8)
$$

and the linear anti-windup compensator as

$$
\dot{\boldsymbol{\xi}}=-\Pi \boldsymbol{\xi}+E \boldsymbol{u}_{\Delta}
$$

where $\beta(s)=a+(1-a) \mathrm{e}^{-b\|s\|^{c}}>0,0<a<1, b>0$, $c>0, k>0, \kappa>0, \lambda_{i}>0, \rho_{i}>0(i=1,2, \cdots, 8) ; K=$ $\operatorname{diag}\left\{K_{1}, K_{2}\right\}, L=\operatorname{diag}\left\{L_{1}, L_{2}\right\}$, and $\Pi=\operatorname{diag}\left\{\Pi_{1}, \Pi_{2}\right\}$ are positive-definite diagonal matrices with $K_{i} \in \mathbb{R}^{3 \times 3}(i=1,2)$, $L_{i} \in \mathbb{R}^{3 \times 3}(i=1,2)$, and $\Pi_{i} \in \mathbb{R}^{3 \times 3}(i=1,2) ; \tanh (\kappa \boldsymbol{s})=$ $\left[\tanh \left(\kappa s_{1}\right), \tanh \left(\kappa s_{2}\right), \cdots, \tanh \left(\kappa s_{6}\right)\right]^{T}$ for $\boldsymbol{s} \in \mathbb{R}^{6} ; \boldsymbol{\xi}$ is the state of the compensator.

Remark 2: In the proposed controller (24), the function $\beta(s)$ has three important properties [35][36]. Firstly, this function is always positive, so it can be viewed as a dynamical gain for the robust control term, such that the stability of the closedloop system is not affected. Secondly, if $s$ increases, $\beta(s)$ approaches to $a$, then $\frac{\gamma}{\beta(s)}$ tends to $\frac{\gamma}{a}$. Thus $\frac{\gamma}{a}$ is larger than $\gamma$, which implies that $\frac{\gamma}{\beta(\boldsymbol{s})}$ increases as $s$ increases, and the convergent rate to $s=0$ will be accelerated. Thirdly, if $s$ decreases, then $\beta(s)$ approaches to 1 , and $\frac{\gamma}{\beta(s)}$ tends to $\gamma$. This implies that, as the system tracking errors approach to zero, $\frac{\gamma}{\beta(s)}$ will be decreased to lower the control effort. Therefore, the function $\beta(s)$ used in this adaptive controller can help the controller to adapt to the variation of $s$ and improve the response performance of the controlled systems.

Theorem 1: Consider the system model (18) of autonomous close-range rendezvous and docking under the Assumptions 1 and 2. If $\boldsymbol{u}_{\Delta}$ is bounded by an unknown constant $\bar{u}_{\Delta}$ such that $\left\|\boldsymbol{u}_{\Delta}\right\| \leq \bar{u}_{\Delta}$, and the tunable parameters satisfy $\frac{\lambda_{\Pi}}{3}-$ $\frac{3 \bar{\lambda}_{L}^{2}}{4 \lambda_{K}}>0$, where $\lambda_{\Pi}$ and $\lambda_{K}$ are minimum eigenvalues of matrices $\Pi$ and $K$, and $\bar{\lambda}_{L}$ is the maximum eigenvalue of the matrix $L$. Then the proposed controller (24) with adaptive laws (25) and suitable tunable parameters can ensure that $\boldsymbol{p}_{e}(t)$ and $\boldsymbol{q}_{e}(t)$ ultimately converge to small neighborhoods of zero, and the estimation errors of unknown parameters are uniformly bounded.

Proof: Substituting the proposed controller into the system model results in the closed-loop system as

$$
\left\{\begin{array}{l}
\dot{\boldsymbol{p}}_{e}=\mathcal{A}_{e} \boldsymbol{p}_{e}+\mathcal{B}_{e} \boldsymbol{q}_{e} \\
\mathcal{M}_{e} \dot{\boldsymbol{s}}+\mathcal{C}_{e} \boldsymbol{s}=\boldsymbol{h}_{\Delta}+\boldsymbol{d}_{e}+E \boldsymbol{u}_{\Delta} \\
-k \mathcal{B}_{e}^{T} \boldsymbol{p}_{e}-K \boldsymbol{s}-L \boldsymbol{\xi}-\frac{\gamma}{\beta(\boldsymbol{s})} \tanh (\kappa \boldsymbol{s})
\end{array}\right.
$$

For the closed-loop system (27), defining the parameter estimation errors as $\tilde{\alpha}_{i}=\hat{\alpha}_{i}-\alpha_{i}(i=1,2, \cdots, 8)$, a candidate Lyapunov function $\mathcal{V}$ is chosen as

$$
\mathcal{V}(t)=\frac{1}{2}\left(k \boldsymbol{p}_{e}^{T} \boldsymbol{p}_{e}+\boldsymbol{s}^{T} \mathcal{M}_{e} \boldsymbol{s}+\boldsymbol{\xi}^{T} \boldsymbol{\xi}+\sum_{i=1}^{8} \frac{\tilde{\alpha}_{i}^{2}}{\lambda_{i}}\right)
$$

Then the time derivative of $\mathcal{V}(t)$ along the state trajectory of the closed-loop system (27) is

$$
\begin{aligned}
\dot{\mathcal{V}}(t)= & k \boldsymbol{p}_{e}^{T} \dot{\boldsymbol{p}}_{e}+\boldsymbol{s}^{T} \mathcal{M}_{e} \dot{\boldsymbol{s}}+\boldsymbol{\xi}^{T} \dot{\boldsymbol{\xi}}+\sum_{i=1}^{8} \frac{\tilde{\alpha}_{i} \dot{\hat{\alpha}}_{i}}{\lambda_{i}} \\
= & k \boldsymbol{p}_{e}^{T} \mathcal{A}_{e} \boldsymbol{p}_{e}+k \boldsymbol{p}_{e}^{T} \mathcal{B}_{e} \boldsymbol{q}_{e}-\boldsymbol{s}^{T} \mathcal{C}_{e} \boldsymbol{s}-\boldsymbol{s}^{T} E \boldsymbol{u}_{\Delta}-\boldsymbol{s}^{T} L \boldsymbol{\xi} \\
& -k \boldsymbol{s}^{T} \mathcal{B}_{e}^{T} \boldsymbol{p}_{e}-\boldsymbol{s}^{T} K \boldsymbol{s}-\frac{\gamma}{\beta(\boldsymbol{s})} \boldsymbol{s}^{T} \tanh (\kappa \boldsymbol{s})+\boldsymbol{s}^{T} \boldsymbol{h}_{\Delta} \\
& +\boldsymbol{s}^{T} \boldsymbol{d}_{e}-\boldsymbol{\xi}^{T} \Pi \boldsymbol{\xi}+\boldsymbol{\xi}^{T} E \boldsymbol{u}_{\Delta}+\sum_{i=1}^{8} \frac{\tilde{\alpha}_{i} \dot{\hat{\alpha}}_{i}}{\lambda_{i}}
\end{aligned}
$$

Since $\boldsymbol{p}_{e}^{T} \mathcal{A}_{e} \boldsymbol{p}_{e}=0$ and $\boldsymbol{s}^{T} \mathcal{C}_{e} \boldsymbol{s}=0$ based on the skewsymmetric matrices $\mathcal{A}_{e}$ and $\mathcal{C}_{e}$, the definition of vector $s$ in 
(22), and the fact $\|s\| \leq \frac{\|s\|_{1}}{\beta(\boldsymbol{s})}$ based on $0<\beta(s)<1$, then the time derivative of $\mathcal{V}(t)$ can be rewritten as

$$
\begin{aligned}
\dot{\mathcal{V}}(t)= & -k \boldsymbol{p}_{e}^{T} \mathcal{B}_{e} \Lambda \boldsymbol{p}_{e}-\boldsymbol{s}^{T} K \boldsymbol{s}-\boldsymbol{\xi}^{T} \Pi \boldsymbol{\xi} \\
& -\boldsymbol{s}^{T} E \boldsymbol{u}_{\Delta}-\boldsymbol{s}^{T} L \boldsymbol{\xi}+\boldsymbol{\xi}^{T} E \boldsymbol{u}_{\Delta}+\boldsymbol{s}^{T} \boldsymbol{h}_{\Delta} \\
& +\boldsymbol{s}^{T} \boldsymbol{d}_{e}-\frac{\gamma}{\beta(\boldsymbol{s})} \boldsymbol{s}^{T} \tanh (\kappa \boldsymbol{s})+\sum_{i=1}^{8} \frac{\tilde{\alpha}_{i} \dot{\hat{\alpha}}_{i}}{\lambda_{i}} \\
\leq & -k \boldsymbol{p}_{e}^{T} \mathcal{B}_{e} \Lambda \boldsymbol{p}_{e}-\boldsymbol{s}^{T} K \boldsymbol{s}-\boldsymbol{\xi}^{T} \Pi \boldsymbol{\xi}+\bar{u}_{\Delta}\|E\|\|\boldsymbol{s}\| \\
& +\bar{u}_{\Delta}\|E\|\|\boldsymbol{\xi}\|+\bar{\lambda}_{L}\|\boldsymbol{s}\|\|\boldsymbol{\xi}\|-\frac{\gamma}{\beta(\boldsymbol{s})} \boldsymbol{s}^{T} \tanh (\kappa \boldsymbol{s}) \\
& +\left(\left\|\boldsymbol{h}_{\Delta}\right\|+\left\|\boldsymbol{d}_{e}\right\|\right) \frac{\|\boldsymbol{s}\|_{1}}{\beta(\boldsymbol{s})}+\sum_{i=1}^{8} \frac{\tilde{\alpha}_{i} \dot{\hat{\alpha}}_{i}}{\lambda_{i}}
\end{aligned}
$$

Since $\|S(\boldsymbol{a})\|=\|\boldsymbol{a}\|$ and $\|R\|=1$, then

$$
\begin{aligned}
\left\|\boldsymbol{h}_{\Delta}\right\|+\left\|\boldsymbol{d}_{e}\right\| \leq & \alpha_{1}\left\|\Lambda\left(\mathcal{A}_{e} \boldsymbol{p}_{e}+\mathcal{B}_{e} \boldsymbol{q}_{e}\right)\right\|+\alpha_{2}\|\boldsymbol{\omega}\|\left\|\Lambda \boldsymbol{p}_{e}\right\|+ \\
& \alpha_{3}\left\|\boldsymbol{\omega}-\boldsymbol{\omega}_{e}\right\|^{2}+\alpha_{4}\left\|\boldsymbol{\omega}_{e}\right\|\left\|\boldsymbol{\omega}-\boldsymbol{\omega}_{e}\right\|+ \\
& \alpha_{5}\|\boldsymbol{\omega}\|\left\|\boldsymbol{\omega}-\boldsymbol{\omega}_{e}\right\|+\alpha_{6}\left\|\boldsymbol{a}_{g}+\boldsymbol{a}_{J_{2}}\right\|+ \\
& \alpha_{7} \frac{3 \mu_{g}}{\|\boldsymbol{r}+\boldsymbol{l}\|^{3}}+\alpha_{8}
\end{aligned}
$$

Because of the facts $0 \leq\left|s_{i}\right|-s_{i} \tanh \left(\kappa s_{i}\right) \leq \frac{\nu}{\kappa}(i=$ $1,2, \cdots, 6)$ with $\nu=0.2785$ and $\tilde{\alpha}_{i} \hat{\alpha}_{i}=\tilde{\alpha}_{i}\left(\tilde{\alpha}_{i}+\alpha_{i}\right)=$ $\frac{1}{2}\left|\tilde{\alpha}_{i}\right|^{2}-\frac{1}{2}\left|\alpha_{i}\right|^{2}+\frac{1}{2}\left|\tilde{\alpha}_{i}+\alpha_{i}\right|^{2}(i=1,2, \cdots, 8)$, then substituting (30) and the proposed adaptive law (25) into (29) results in

$$
\begin{aligned}
\dot{\mathcal{V}}(t) \leq & -k \lambda_{\Lambda}\left\|\boldsymbol{p}_{e}\right\|^{2}-\frac{\lambda_{K}}{3}\|\boldsymbol{s}\|^{2}-\frac{\lambda_{\Pi}}{3}\|\boldsymbol{\xi}\|^{2} \\
& -\left(\frac{\lambda_{\Pi}}{3}-\frac{3 \bar{\lambda}_{L}^{2}}{4 \lambda_{K}}\right)\|\boldsymbol{\xi}\|^{2}+\frac{3 \bar{u}_{\Delta}^{2}\|E\|^{2}}{4 \lambda_{K}}+\frac{3 \bar{u}_{\Delta}^{2}\|E\|^{2}}{4 \lambda_{\Pi}} \\
& -\sum_{i=1}^{8} \frac{\rho_{i}}{2 \lambda_{i}}\left|\tilde{\alpha}_{i}\right|^{2}+\sum_{i=1}^{8} \frac{\rho_{i}}{2 \lambda_{i}}\left|\alpha_{i}\right|^{2}+\frac{6 \nu}{\kappa} \\
\leq & -\delta_{0}\|\boldsymbol{z}\|^{2}+\epsilon
\end{aligned}
$$

where $\boldsymbol{z} \triangleq\left[\boldsymbol{p}_{e}^{T}, \boldsymbol{s}^{T}, \boldsymbol{\xi}^{T}, \tilde{\alpha}_{1}, \tilde{\alpha}_{2}, \cdots, \tilde{\alpha}_{8}\right]^{T} ; \quad \delta_{0} \triangleq$ $\min \left\{k \lambda_{\Lambda}, \frac{\lambda_{K}}{3}, \frac{\lambda_{\Pi}}{3}, \frac{\rho_{i}}{2 \lambda_{i}}(i \quad=\quad 1,2, \cdots, 8)\right\} ; \quad \lambda_{\Lambda} \triangleq$ $\min \left\{\lambda_{m}\left(\Lambda_{1}\right), \frac{1}{4} \lambda_{m}\left(\Lambda_{2}\right)\right\} ; \lambda_{m}\left(\Lambda_{i}\right)$ is the minimum eigenvalue of $\Lambda_{i}(i=1,2) ; \epsilon \triangleq \frac{3 \bar{u}_{\Delta}^{2}\|E\|^{2}}{4 \lambda_{K}}+\frac{3 \bar{u}_{\Delta}^{2}\|E\|^{2}}{4 \lambda_{\Pi}}+\sum_{i=1}^{8} \frac{\rho_{i}}{2 \lambda_{i}}\left|\alpha_{i}\right|^{2}+\frac{6 \nu}{\kappa}$. According to the definition of $\mathcal{V}(t)$ in (28), one has

$$
\delta_{1}\|\boldsymbol{z}\|^{2} \leq \mathcal{V}(t) \leq \delta_{2}\|\boldsymbol{z}\|^{2}
$$

where $\delta_{1} \triangleq \min \left\{\frac{1}{2}, \frac{k}{2}, \frac{\lambda_{\mathcal{M}_{e}}}{2}, \frac{1}{2 \lambda_{i}}(i=1,2, \cdots, 8)\right\}, \delta_{2} \triangleq$ $\max \left\{\frac{1}{2}, \frac{k}{2}, \frac{\bar{\lambda}_{\mathcal{M}_{e}}}{2}, \frac{1}{2 \lambda_{i}}(i=1,2, \cdots, 8)\right\}$, and $\lambda_{\mathcal{M}_{e}}$ and $\bar{\lambda}_{\mathcal{M}_{e}}$ are minimum and maximum eigenvalues for $\mathcal{M}_{e}$, respectively. Thus, one has $\dot{\mathcal{V}}(t) \leq-\frac{\delta_{0}}{\delta_{2}} \mathcal{V}(t)+\epsilon$, and its solution satisfies $\mathcal{V}(t) \leq\left[\mathcal{V}(0)-\frac{\epsilon \delta_{2}}{\delta_{0}}\right] \mathrm{e}^{-\frac{\delta_{0}}{\delta_{2}} t}+\frac{\epsilon \delta_{2}}{\delta_{0}}$. This means $\|\boldsymbol{z}\| \leq$ $\sqrt{\frac{1}{\delta_{1}}\left[\mathcal{V}(0)-\frac{\epsilon \delta_{2}}{\delta_{0}}\right] \mathrm{e}^{-\frac{\delta_{0}}{\delta_{2}} t}+\frac{\epsilon \delta_{2}}{\delta_{1} \delta_{0}}}$. Thus $\boldsymbol{p}_{e}, \boldsymbol{s}, \boldsymbol{\xi}$, and $\tilde{\alpha}_{i}(i=$ $1,2, \cdots, 8)$ are ultimately bounded. Since $\left\|\boldsymbol{p}_{e}\right\| \leq\|\boldsymbol{z}\|$, then $\lim _{t \rightarrow \infty}\left\|\boldsymbol{p}_{e}(t)\right\| \leq \lim _{t \rightarrow \infty}\|\boldsymbol{z}(t)\| \leq \sqrt{\frac{\epsilon \delta_{2}}{\delta_{1} \delta_{0}}}$. Furthermore, since $\boldsymbol{q}_{e}=\boldsymbol{s}-\Lambda \boldsymbol{p}_{e},\|\boldsymbol{s}\| \leq\|\boldsymbol{z}\|$, and $\left\|\underline{\underline{\boldsymbol{p}_{e}} \|} \leq\right\| \boldsymbol{z} \|$, then one has $\lim _{t \rightarrow \infty}\left\|\boldsymbol{q}_{e}(t)\right\| \leq\left(1+\bar{\lambda}_{\Lambda}\right) \sqrt{\frac{\epsilon \delta_{2}}{\delta_{1} \delta_{0}}}$ with the maximum eigenvalue $\bar{\lambda}_{\Lambda}$ of the matrix $\Lambda$. Therefore, $\boldsymbol{p}_{e}(t)$ and $\boldsymbol{q}_{e}(t)$ ultimately converge to small neighborhoods of zero, and compensator state $\boldsymbol{\xi}$ and the parameter estimation errors $\tilde{\alpha}_{i}(i=1,2, \cdots, 8)$ are uniformly bounded.

Remark 3: The proposed controller (24) has a typical statefeedback control structure with the proportional-derivative feedback terms $-\left(k \mathcal{B}_{e}^{T}+K \Lambda\right) \boldsymbol{p}_{e}-K \boldsymbol{q}_{e}$, the feed-forward term $-\boldsymbol{h}_{0}$, saturation compensation term $-L \boldsymbol{\xi}$, and the robust adaptive control term $-\frac{\gamma}{\beta(s)} \tanh (\kappa s)$, where feedback terms have three tunable parameters, $k, K$, and $\Lambda$, to set the transient and steady-state response performances of the closed-loop system; feed-forward term is employed to cancel the known part of the system model; the robust adaptive control term is used to suppress the model uncertainties. In particular, the tunable parameters $K$ and $\Lambda$ can be chosen independently for the relative translational and relative rotational motions, such as $K_{1}$ and $\Lambda_{1}$ for relative position subsystem, $K_{2}$ and $\Lambda_{2}$ for relative attitude subsystem.

\section{Simulation Example}

A numerical simulation is performed for a rendezvous and docking mission, where the chaser tracks the docking port fixed on the tumbling target. The physical parameters of two spacecraft are [18] $m=58.2(\mathrm{~kg}), m_{t}=5425.6(\mathrm{~kg})$,

$$
\begin{gathered}
J=\left[\begin{array}{ccc}
38 & -2.5 & -5.5 \\
-2.5 & 44 & -2.7 \\
-5.5 & -2.7 & 36
\end{array}\right]\left(\mathrm{kgm}^{2}\right), \\
J_{t}=\left[\begin{array}{ccc}
3336 & -135.4 & -154.2 \\
-135.4 & 3184 & -148.5 \\
-154.2 & -148.5 & 2423
\end{array}\right]\left(\mathrm{kgm}^{2}\right) .
\end{gathered}
$$

The known parameters about chaser are $m_{0}=60(\mathrm{~kg})$, $J_{0}=\operatorname{diag}\{35,40,40\}\left(\mathrm{kgm}^{2}\right)$. Since the distance between two spacecraft is much smaller than their orbital radius, then the frequency of the external disturbances for two spacecraft is set as $\omega_{o}=\sqrt{\mu_{g} /\left\|R_{c}(\boldsymbol{r}+\boldsymbol{l})\right\|^{3}}$. Thus the external disturbances in simulations are assumed as

$$
\begin{aligned}
& \boldsymbol{w}=\left[\begin{array}{l}
3-2 \sin \left(\omega_{o} t\right)-4 \cos \left(\omega_{o} t\right) \\
3-3 \sin \left(\omega_{o} t\right)+2 \cos \left(\omega_{o} t\right) \\
2+4 \sin \left(\omega_{o} t\right)+2 \cos \left(\omega_{o} t\right)
\end{array}\right] \times 10^{-3}+\text { Rand } \times 10^{-4}(\mathrm{~N}), \\
& \boldsymbol{\delta}=\left[\begin{array}{l}
6-4 \sin \left(\omega_{o} t\right)+5 \cos \left(\omega_{o} t\right) \\
7+5 \sin \left(\omega_{o} t\right)-4 \cos \left(\omega_{o} t\right) \\
4-3 \sin \left(\omega_{o} t\right)+3 \cos \left(\omega_{o} t\right)
\end{array}\right] \times 10^{-4}+\text { Rand } \times 10^{-5}(\mathrm{Nm}),
\end{aligned}
$$

$\boldsymbol{w}_{t}=100 \boldsymbol{w}(\mathrm{N})$, and $\boldsymbol{\delta}_{t}=100 \boldsymbol{\delta}(\mathrm{Nm})$, where Rand denotes the random Gaussian white noises with zero mean.

The initial conditions for chaser motion and relative motion are respectively set as $\boldsymbol{r}(0)=[1,1,1]^{T} \times 7.078 \times 10^{6}(\mathrm{~m})$, $\boldsymbol{v}(0)=[2,3,-2]^{T} \times 10^{3}(\mathrm{~m} / \mathrm{s}), \boldsymbol{\sigma}(0)=\mathbf{0}, \boldsymbol{\omega}(0)=\mathbf{0}(\mathrm{rad} / \mathrm{s})$, $\boldsymbol{r}_{e}(0)=[-5,5,-5]^{T}(\mathrm{~m}), \boldsymbol{v}_{e}(0)=[-0.3,-0.2,0.2]^{T}(\mathrm{~m} / \mathrm{s})$, $\boldsymbol{\sigma}_{e}(0)=[0.5,-0.6,0.7]^{T}, \boldsymbol{\omega}_{e}(0)=[0.02,0.02,0.02]^{T}$. The known vectors are $\boldsymbol{p}_{d}=[0,5,0]^{T}(\mathrm{~m})$ and $\boldsymbol{l}=[0.5,0,0]^{T}(\mathrm{~m})$. The lower and upper limits of control inputs are $\tau_{i_{\min }}=1(\mathrm{Nm})$, $\tau_{i_{\max }}=0.3(\mathrm{Nm}), f_{i_{\text {min }}}=3(\mathrm{~N}), f_{i_{\text {max }}}=9(\mathrm{~N})$. The controller parameters are $\Lambda=0.001 I_{6}, K_{1}=400 I_{3}, K_{2}=40 I_{3}, L=$ $0.005 I_{6}, \Pi=0.5 I_{6}, k=0.001, a=0.2, b=0.5, c=2$, $\kappa=20, \lambda_{i}=0.2, \rho_{i}=0.5(i=1,2, \cdots, 8)$.

Figures 2-5 show the simulation results based on the proposed controller. Figures 2 and 3 give the relative pose results, 
where the relative position and relative attitude converge to the neighborhoods of zero in 30(s) with steady-state high precision. Figure 4 gives the time history of control inputs with smaller control chattering in the steady-state phase. Figure 5 implies that all estimations are always bounded. These results imply that the proposed controller (24) can ensure the relative position tracking and attitude synchronization mission with acceptable performance for close-range rendezvous and docking missions. Furthermore, to validate the advantages of the tuning function $\beta(s)$ in the proposed controller (24), the simulation results based on the non-tuning function version of (24) are shown in Figures 6-9. From the comparisons between two kinds of controlled systems, the proposed tuning functionbased controller has better performance with faster transient response time and higher steady-state precision and stronger anti-disturbance ability. Furthermore, to show the effectiveness of the saturation compensator, the proposed controller without the compensator is used to conduct the simulation again, and the results are given in Figures 10-13. It is seen that the curves of the system states in Figures 10 and 11 are slightly different in the transient response process with respect to the results in Figures 2 and 3, but the control effort in Figure 12 is clearly larger than the ones in Figure 13, and the magnitude of control inputs clearly exceeds the prescribed nonsymmetric limitation. Thus the proposed compensator-based adaptive controller has better control performance with respect to the controller without the saturation compensator.
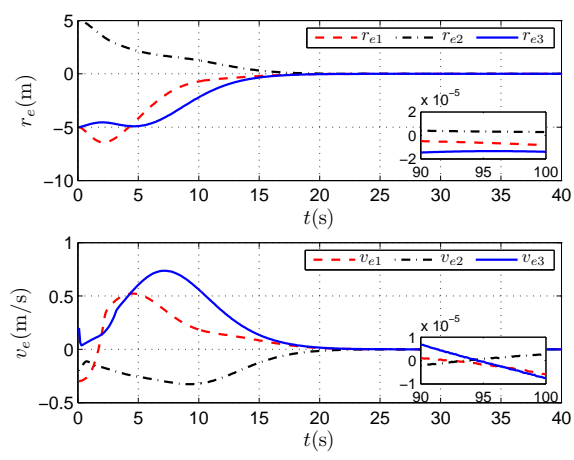

Fig. 2. Relative position and velocity under (24).
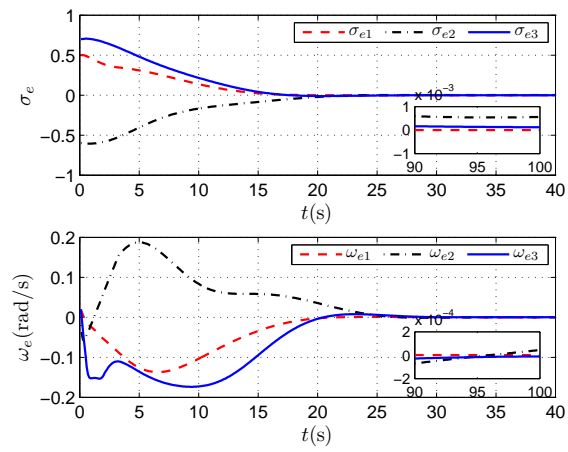

Fig. 3. Relative attitude and angular velocity under (24).
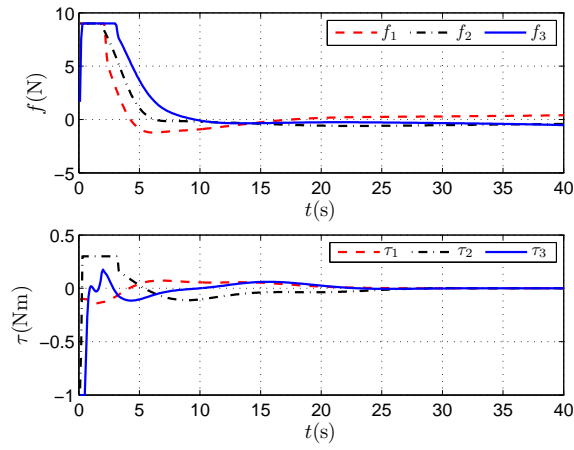

Fig. 4. Control forces and torques under (24).
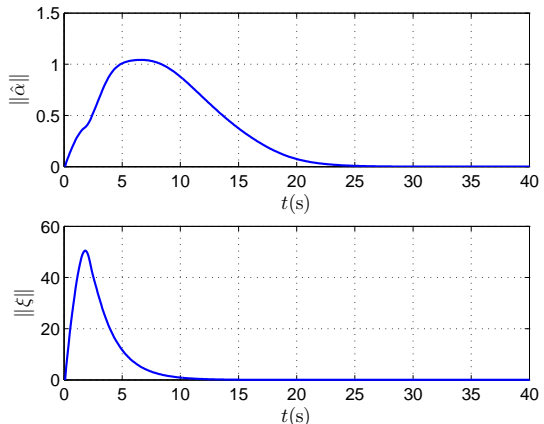

Fig. 5. Norms of estimations and compensator states under (24).
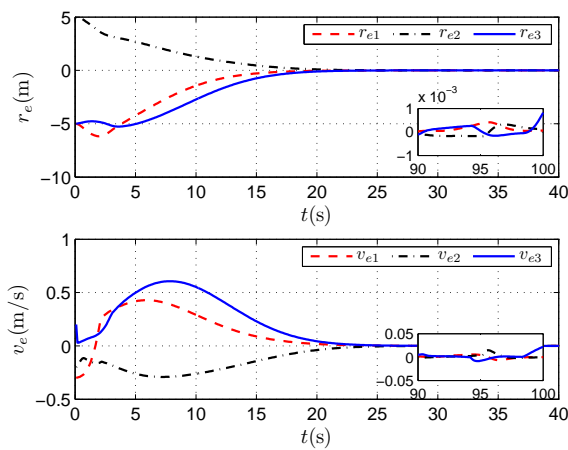

Fig. 6. Relative position and velocity under (24) without $\beta(s)$.
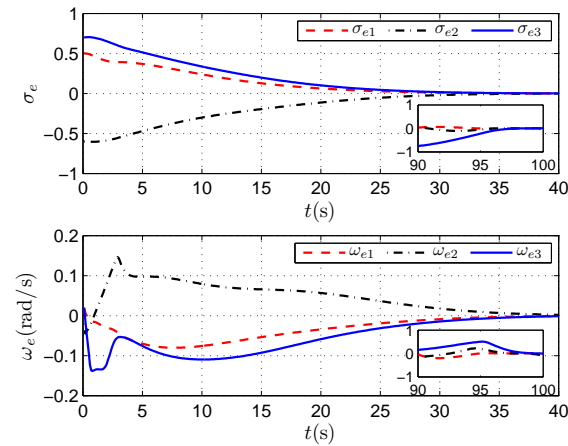

Fig. 7. Relative attitude and angular velocity under (24) without $\beta(s)$. 

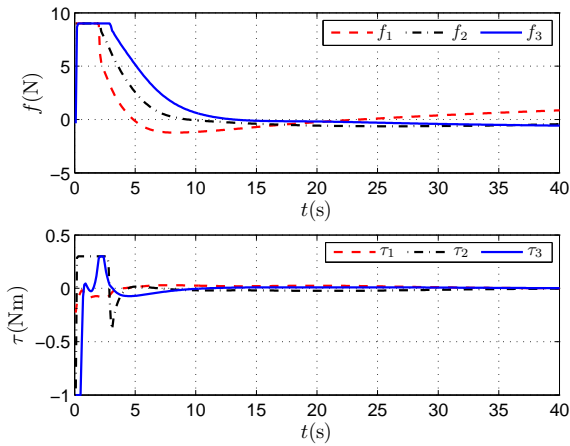

Fig. 8. Control forces and torques under (24) without $\beta(s)$.
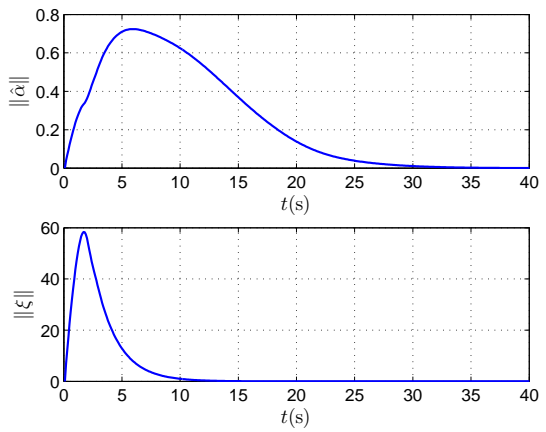

Fig. 9. Norms of estimations and compensator states under (24) without $\beta(\boldsymbol{s})$.
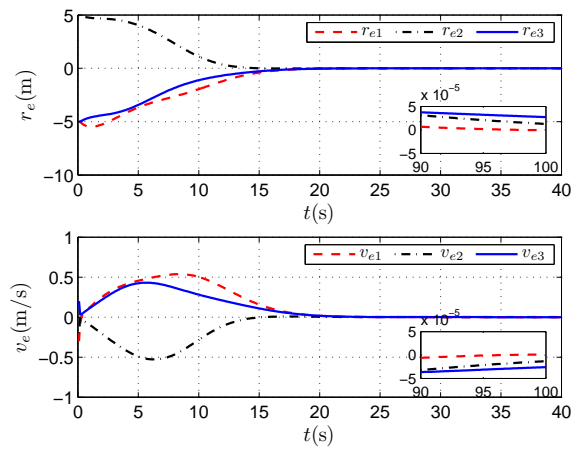

Fig. 10. Relative position and velocity under (24) without compensator (26).
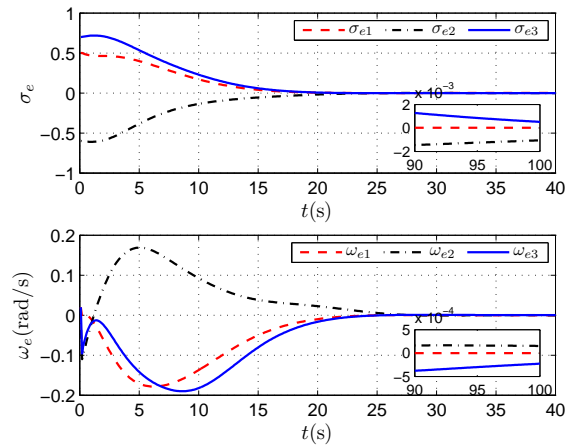

Fig. 11. Relative attitude and angular velocity under (24) without compensator (26).
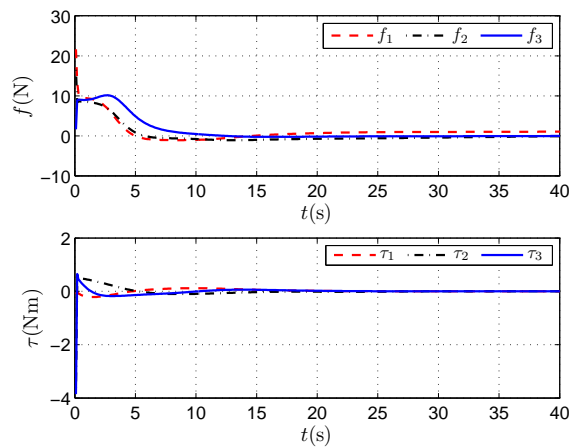

Fig. 12. Control forces and torques under (24) without compensator (26).
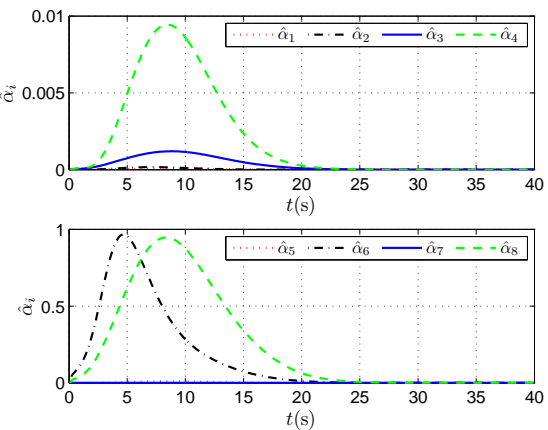

Fig. 13. Adaptive estimations under (24) without compensator (26).

\section{CONCLUSIONS}

A model-based adaptive sliding mode relative pose motion controller has been developed for the spacecraft close-range rendezvous and docking missions. A relative pose dynamics between the docking ports of two spacecraft are formulated in the chaser's body-fixed coordinate frame to reflect the complicated couplings in the model, and the proposed controller has classical state-feedback control structure with less online estimations to suppress the model uncertainties and guarantee the response performance. The nonsymmetric saturation effect of the control inputs is compensated by a linear anti-windup auxiliary system in the control system. Theoretical analysis and simulation results imply that the proposed controlled system can ensure the relative motion states ultimately converging to small neighborhoods of zero. The future works will focus on the advanced control approaches for the proposed relative motion model subject to multiple constraints.

\section{REFERENCES}

[1] J. L. Goodman, "History of space shuttle rendezvous and proximity operations," J. Spacecraft Rockets, vol. 43, no. 5, pp. 944-959, 2006.

[2] C. A. Kluever, "Feedback control for spacecraft rendezvous and docking," J. Guid. Control Dyn., vol. 22, no. 4, pp. 609-611, Jul./Aug. 1999.

[3] Y. Wang, S. Xu, "Stabilization of coupled orbit-attitude dynamics about an asteroid utilizing Hamiltonian structure," Astrodyn., vol. 2, no. 1, pp. 53-67, Mar. 2018.

[4] K. Subbarao and S. Welsh, "Noninear control of motion synchronization for satellite proximity operations," J. Guid. Control Dyn., vol. 31, no. 5, pp. 1284-1294, Sep./Oct. 2008.

[5] D. Lee and G. Vukovich, "Adaptive sliding mode control for spacecraft body-fixed hovering in the proximity of an asteroid," Aerosp. Sci. Technol., vol. 46, pp. 471-483, Oct. 2015. 
[6] Y. Ikeda, T. Kida and T. Nagashio, "Stabilizing nonlinear adaptive control of spacecraft before and after capture," Trans. Japan Soc. Aero. Space Sci., vol. 59, no. 1, pp. 1-9, Jan. 2016.

[7] M. Xin and H. Pan, "Indirect robust control of spacecraft via optimal control solution," IEEE Trans. Aerosp. Electron. Syst., vol. 48, no. 2, pp. 1798-1809, Apr. 2012.

[8] H. Yoon, Y. Eun, and C. Park, "Adaptive tracking control of spacecraft relative motion with mass and thruster uncertainties," Aerosp. Sci. Technol., vol. 34, pp. 75-83, Apr. 2014.

[9] S. B. McCamish, M. Romano, and X. Yun "Autonomous distributed control of simulaneous multiple spacecraft proximity maneuvers," IEEE Trans. Autom. Sci. Eng., vol. 7, no.3, pp. 630-643, Jul. 2010.

[10] F. Zhang and G. Duan, "Integrated relative position and attitude control of spacecraft in proximity operation missions," Int. J. Autom. Comput., vol. 9, no. 4, pp. 342-351, Aug. 2012.

[11] L. Sun and W. Huo, "6-DOF integrated adaptive backstepping control for spacecraft proximity operations," IEEE Trans. Aerosp. Electron. Syst., vol. 51, no. 3, pp. 2433-2443, Jul. 2015.

[12] L. Sun, W. He, and C. Sun, "Adaptive fuzzy relative pose control of spacecraft during rendezvous and proximity operations," IEEE Trans. Fuzzy Syst., vol. 26, no. 6, pp. 3440-3451, Dec. 2018.

[13] L. Sun and Z. Zheng, "Adaptive relative pose control of spacecraft with model couplings and uncertainties," Acta Astronautica, vol. 143, pp. 29-36, Feb. 2018.

[14] G. Di Mauro, M. Schlotterer, S. Theil, and M. Lavagna, "Nonlinear control for proximity operations based on differential algebra," J. Guid. Control Dyn., vol. 38, no. 11, pp. 2173-2187, Nov. 2015.

[15] N. Filipe and P. Tsiotras, "Adaptive position and attitude-tracking controller for satellite proximity operations using dual quaternions," $J$. Guid. Control Dyn., vol. 38, no. 4, pp. 566-577, Apr. 2015.

[16] H. Dong, Q. Hu, and M. R. Akella, "Dual-quaternion-based spacecraft autonomous rendezvous and docking under six-degree-of-freedom motion constraints," J. Guid. Control Dyn., vol. 41, no. 5, pp. 1150-1162, May 2018.

[17] L. Sun, W. Huo, and Z. Jiao, "Robust nonlinear adaptive relative pose control for cooperative spacecraft during rendezvous and proximity operations," IEEE Trans. Control Syst. Technol., vol. 25, no. 5, pp. 18401847, Sep. 2017.

[18] L. Sun, W. Huo, and Z. Jiao, "Disturbance-observer-based robust relative pose control for spacecraft rendezvous and proximity operations under input saturation," IEEE Trans. Aerosp. Electron. Syst., vol. 54, no. 4, pp. 1605-1617, Aug. 2018

[19] F. Zhang, "Robust integrated transalational and rotational control for spacecraft rendezvous in unstructured environments," Trans. Inst. Measurement Control, vol. 40, no. 11, pp. 3293-3313, Jul. 2018.

[20] S. He and D. Lin, "Reliable spacecraft rendezvous without velocity measurement," Acta Astronaut., vol. 144, pp. 52-60, Mar. 2018.

[21] D. Lee and G. Vukovich, "Robust adaptive terminal sliding mode control on SE(3) for autonomous spacecraft rendezvous and docking," Nonlinear Dyn., vol. 83, no. 4, pp. 2263-2279, Mar. 2016.

[22] M. Leomanni, E. Rogers, and S. B. Gabriel, "Explicit model predicitve control approach for low-thrust spacecraft proximity operations" J. Guid. Control Dyn., vol. 37, no. 6, pp. 1780-1790, Nov. 2014.

[23] S. Di Cairano, H. Park, and I. Kolmanovsky, "Model predictive control approach for guidance of spacecraft rendezvous and proximity maneuvering," Int. J. Robust Nonlinear Control, vol. 22, no. 12, pp. 1398-1427, Aug. 2010.

[24] A. Weiss, M. Baldwin, R. S. Erwin, and I. Kolmanovsky, "Model predictive control for spacecraft rendezvous and docking: startegies for handling constraints and case studies," IEEE Trans. Control Syst. Technol., vol. 23, no. 4, pp. 1638-1647, Jul. 2015.

[25] S. Ulrich, A. Saenz-Otero, and I. Barkana, "Passivity-based adaptive control of robotic spacecraft for proximity operations uncer uncertainties," J. Guid. Control Dyn., vol. 39, no. 6, pp. 1441-U340, Jun. 2016.

[26] J. Long and F. Wu, "Iterative-learning-control-based tracking for asteroid close-proximity operations," J. Guid. Control Dyn., vol. 42, no. 5, pp. 1195-1203, May 2019.

[27] Q. Hu, X. Shao, and W.-H. Chen, "Robust fault-tolerant tracking control for spacecraft proximity operations using time-varying sliding mode," IEEE Trans. Aerosp. Electron. Syst., vol. 54, no. 1, pp. 2-17, Feb. 2018

[28] H. Dong, Q. Hu, Y. Liu, and M. R. Akella, "Adaptive pose tracking control for spacecraft proximity operations under motion constraints," J. Guid. Control Dyn., vol. 42, no. 10, pp. 2258-2271, Jul. 2019.

[29] N. Filipe, A. Valverde, and P. Tsiotras, "Pose tracking without linear and angular-velocity feedback using dual quatenions," IEEE Trans. Aerosp. Electron. Syst., vol. 52, no. 1, pp. 411-422, Feb. 2016.
[30] Q. Dang, H. Gui, and H. Wen, "Dual-quaternion-based spacecraft pose tracking with a global exponential velocity observer," J. Guid. Control Dyn., vol. 42, no. 9, pp. 2106-2115, Sep. 2019.

[31] Y. Deng and Z. Wang, "Modeling and control for spacecraft relative pose motion by using twistor representation," J. Guid. Control Dyn., vol. 39, no. 5, pp. 1144-1151, May 2016.

[32] J. Yang and E. Stoll, "Adaptive sliding mode control for spacecraft proximity operations based on dual quaternions," J. Guid. Control Dyn., vol. 42, no. 11, pp. 2356-2368, Jul. 2019.

[33] H. Pan, V. Kapila, "Adaptive nonlinear control for spacecraft formation flying with coupled translational and attitude dynamics," Proc. 40th IEEE Conf. Decision Control, IEEE, vol. 3, pp. 2057-2062, 2001.

[34] H. Schaub and J. L. Junkins, Analytical Mechanics of Space Systems, Reston: AIAA, 2003

[35] R. Rascon, O. Penaloza-Mejia, J. Castro, "Improving first order sliding mode control on second order mechanical systems," European J. Control, vol. 29, pp. 74-80, May 2016.

[36] Q. Chen, S. Xie, M. Sun, and X. He, "Adaptive nonsingular fixedtime attitude stabilization of uncertain spacecraft," IEEE Trans. Aerosp. Electron. Syst., vol. 54, no. 6, pp.2937-2950, Dec. 2018.

[37] J. Slotine and W. Li, Applied Nonlinear Control, Prentice Hall, New Jersey, 1991.

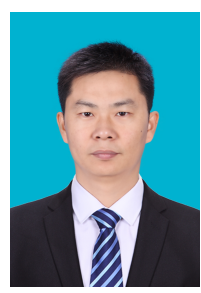

Liang Sun received the M.Sc. and Ph.D. degrees in control theory and control engineering from Beihang University, China, in 2011 and 2016, respectively. He was a Postdoctoral Fellow in mechanical engineering with Beihang University from 2015 to 2017, and a Visiting Scholar with Loughborough University, UK, from 2019 to 2020 . He joined University of Science and Technology Beijing as an Associate Professor in 2017. His current research interests include nonlinear mechanical systems control, aerospace control and intelligent control.

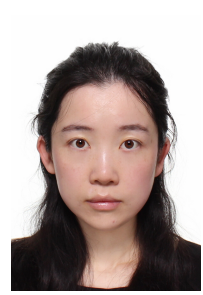

Jingjing Jiang received the B.E. Degree in Electrical and Electronic Engineering from the University of Birmingham, UK, and the Harbin Institute of Technology, China, in 2010, the M.Sc. degree in Control Systems from Imperial College London, UK, in 2011, and the Ph.D degree from Imperial College London, in 2016. She carried out research as part of the Control and Power Group at Imperial College and joined Loughborough University as a Lecturer in September 2018. Her current research interests include driver assistance control and autonomous vehicle control design, control design of systems with constraints and human-in-the-loop. 